\title{
ESTIMAÇÃO EM CATEGORIAS DOS DESCRITORES DA DOR PÓS-OPERATÓRIA*
}

\author{
Lilian Varanda Pereira** \\ Fátima Aparecida Emm Faleiros Sousa***
}

PEREIRA, L.V.; SOUSA, F.A.E.F. Estimação em categorias dos descritores da dor pós-operatória. Rev.latinoam.enfermagem, Ribeirão Preto, v. 6, n. 4, p. 41-48, outubro 1998.

Neste estudo investigamos a linguagem utilizada por enfermeiros na caracterização da dor pós-operatória. Foi elaborado um instrumento contendo, inicialmente, 308 descritores de dor. Este foi analisado quanto à validade aparente e de conteúdo. Após essa análise, com 119 descritores, aplicamos o instrumento para 116 enfermeiros. Foi utilizado o método de estimação em categorias, com escores variando de 1 a 7. Foram calculados a média aritmética e o desvio-padrão dos escores obtidos. Os descritores de maior atribuição, em ordem decrescente, foram: intensa, forte, aguda insuportável e contínua e os de menor atribuição foram: circulante, assaltante, circular, vagarosa e formidável.

UNITERMOS: estimação em categorias, dor pós-operatória, mensuração da dor

\section{INTRODUÇÃO}

Nas últimas décadas, mediante os avanços ocorridos nos estudos pertinentes à compreensão do fenômeno álgico, muito tem sido discutido sobre a necessidade de se obter instrumentos que possibilitem mensurar a dor, experiência complexa, subjetiva e multidimensional.

Como tal, compreendemos que, a descrição verbal da intensidade da dor e de suas qualidades, feita pelo próprio indivíduo que a sente, é de fundamental importância à sua mensuração. Evidentemente, conhecer a linguagem utilizada nessa descrição (os descritores de dor) e compreender aquilo que está sendo transmitido por seu intermédio é essencial para os profissionais da área que almejam o seu controle.

A dor é a complicação mais freqüente no período pós-operatório e a descrição da mesma é objeto de nosso estudo. Sua intensidade depende da influência de fatores fisiológicos, como a extensão do trauma durante a intervenção, a habilidade técnica do cirurgião, as doenças prévias, o local e o tipo da incisão; de fatores psicológicos (ansiedade, depressão, medo, dentre outros) bem como de fatores culturais do paciente. (FORTUNA \& FORTUNA, 1987; EISENACH, 1993; READY \& EDWARDS, 1995).

A ocorrência dessa dor, como também a sua persistência, pode gerar ansiedade, angústia, perturbações cardiovasculares, respiratórias e síndromes ainda pouco exploradas, como a síndrome neuro-endócrina pósoperatória (CONSTANT \& BONNET, 1993). Tais ocorrências prejudicam a recuperação do paciente, prolongam sua estadia no hospital, elevam o custo do tratamento e colocam em risco a sua própria vida, preço alto, que muitas vezes é pago desnecessariamente. Segundo EISENACH (1993) e READY \& EDWARDS (1995), o insucesso em proporcionar alívio adequado à dor no pós-operatório é atribuído, principalmente, à dificuldade em se modificar intervenções inadequadas decorrentes da falta de conhecimento dos profissionais da área, com vistas à avaliação e mensuração da dor, às doses efetivas dos analgésicos, especialmente dos narcóticos, e ao tempo de ação e efeitos colaterais dessas drogas.

Revisando a literatura específica, levantamos estudos nos quais foram avaliadas as atitudes de médicos

\footnotetext{
* O presente trabalho constitui parte da Dissertação de Mestrado, defendida em 1996, pela primeira autora, Docente do Departamento de Enfermagem na Assistência Hospitalar do Centro de Graduação em Enfermagem da FMTM

Endereço para correspondência:

Centro de Graduação em Enfermagem da Faculdade de Medicina do Triângulo Mineiro

Praça Manoel Terra - S/N

38040060 - Uberaba - MG

** Professor Assistente do Departamento de Enfermagem na Assistência Hospitalar do Centro de Graduação em Enfermagem da FMTM

*** Professor Doutor do Departamento de Enfermagem Geral e Especializada da EERP- USP, orientador da dissertação
} 
e de enfermeiros frente à dor pós-operatória, sendo detectado sofrimento desnecessário dos pacientes. Esse sofrimento foi atribuído a duas causas principais, sendo a primeira, erros na prescrição e administração de drogas analgésicas devido a "medos" infundados quanto ao desenvolvimento de vício e depressão respiratória mediante o uso de narcóticos, falta de conhecimento sobre possíveis efeitos colaterais dessas drogas, associação dessas a outros fármacos e tempo de ação das mesmas e, a segunda, avaliações inadequadas, muitas vezes influenciadas pelos fatores culturais, religiosos, experiência pessoal com dor intensa e experiência profissional de médicos e enfermeiros( MARKS \& SACHAR, 1973; McCAFFERY \& HART, 1976; MELZAC et al., 1982; WEIS et al., 1983; CARTWRIGHT, 1985; OWEN et al., 1990; PIMENTA et al., 1992; DONOVAN et al.1994; HARDWICK \& DURDEY, 1994).

No que se refere à influência dos fatores culturais na avaliação da dor feita por tais profissionais citamos o estudo de DAVITZ \& PENDLENTON (1969), realizado com 130 enfermeiros de diferentes grupos culturais (32 coreanos, 30 tailandeses, 23 porto-riquenhos, 20 norteamericanos brancos e 25 norte-americanos negros); ressaltaram que as inferências de dor diferiram entre os profissionais, mostrando médias (obtidas através dos escores atribuídos em uma escala de 0 a 6 pontos) maiores para os enfermeiros porto-riquenhos $(\mathrm{M}=4,42 ; \mathrm{DP}=0,57)$ e menores para os enfermeiros norte-americanos negros $(\mathrm{M}=3,73 ; \mathrm{DP}=0,35)$. (ver também MARTIN \& BELCHER, 1986; GREENWALD, 1991).

Num outro exemplo, McCAFFERY (1980) comenta que o fato de se considerar, culturalmente, os homens mais tolerantes à dor do que as mulheres pode levar profissionais da saúde a serem mais sensíveis em oferecer alívio a esses pacientes do que àqueles do sexo feminino, por considerarem que as mulheres exacerbam suas queixas de dor.

HOLM et al. (1989) mostraram em seu estudo que experiência pessoal com dor intensa e religião de 134 enfermeiros foram variáveis importantes na avaliação da dor do paciente. Os resultados alertaram para o fato de que os profissionais que tiveram experiências dolorosas anteriormente, mostravam-se mais atenciosos aos pacientes com dor e os enfermeiros que possuíam uma religião definida, pareciam atribuir menor grau de intensidade de dor física nos julgamentos da mesma. Para outras informações a respeito da influência de características do observador, na avaliação da dor do paciente, ver LENBURG et al. (1970); TESKE et al. (1983); CAMP \& O'SULLIVAN (1987).

Adicionado a isso, CHOINIÈRE et al. (1990), apontaram para a experiência profissional dos enfermeiros como fator de interferência na avaliação da intensidade da dor de pacientes submetidos a procedimentos terapêuticos dolorosos. Os profissionais menos experientes, a despeito dos procedimentos realizados, superestimaram a dor dos pacientes, enquanto outros, mais experientes, subestimaram sua intensidade (ver também ZALON, 1993; EISENACH, 1993).

Nesse contexto, onde tão complexa experiência tem sido avaliada e conseqüentemente tratada muito mais pelos pressupostos dos profissionais da área do que por aquilo que o paciente de fato está sentindo, compreendemos que, além de conhecimentos básicos sobre o uso de analgésicos, necessitamos de instrumentos fidedignos que nos possibilitem mensurá-la qualitativa e quantitativamente. Assim, poderemos conhecer a natureza e a intensidade da dor sentida, ou seja, se está acima do limiar, causando desconforto ao paciente, de tal forma a impedir suas atividades físicas e intelectuais normais.

Referindo-nos à dor pós-operatória, observamos que os instrumentos mais freqüentemente utilizados à sua mensuração são ordinais, podendo ser de dois tipos: unidimensionais e multidimensionais. Dentre os unidimensionais relevam-se as escalas numéricas, verbais e visuais analógicas; dentre os multidimensionais relevamse aqueles que utilizam a abordagem de MELZACK \& TORGERSON (1971), tal como o Questionário para Dor de McGill (MELZACK, 1975). Tais autores investigaram as palavras (descritores de dor), utilizadas no cotidiano clínico para descrever a dor sentida e defenderam a idéia de que estas poderiam representar as dimensões sensorial, afetiva e avaliativa da experiência dolorosa e, logo, serem fundamentais na elaboração de instrumentos multidimensionais, necessários à mensuração da dor em mais de duas dimensões. O MPQ, primeiro instrumento elaborado a partir das palavras selecionadas por MELZACK \& TORGERSON (1971) tem sido utilizado em inúmeras pesquisas, em diversos idiomas como, o italiano (MAIANI \& SANAVIO, 1985; De BENEDITTIS et al., 1988), o finlandês (PÖNTINEN \& KETOVUORI, 1983), o espanhol (LÓPES et al., 1990), o norueguês (STRAND \& WISNES, 1991), o alemão (RADVILA et al., 1987), o holandês (VANDERIET et al., 1987), o arábico (HARRISON, 1988) e o francês (BOUREAU et al., 1992), para mensurar a dor clínica, aguda e crônica; testar a eficácia das técnicas analgésicas empregadas e discriminar a dor decorrente de diversas síndromes dolorosas. Embora mais freqüentemente utilizado para a mensuração da dor crônica o MPQ também tem sido utilizado no pós-operatório, como podemos observar nos trabalhos de MELZACK et al., (1982); READING (1982); KIM et al. (1995); MELZACK et al. (1987); KATZ et al. (1994); STEIN et al. (1991); BOULANGER et al. (1993); SCHULTE-STEINBERG et al. (1995); THOMAS et al. (1995).

Compreendendo a dor como uma experiência multidimensional passível de ser mensurada propomo-nos, com esse estudo, colaborar com a mensuração da dor 
clínica aguda investigando a linguagem utilizada na caracterização da dor pós-operatória em nosso país. Temos como objetivos:

1. Construir um instrumento, de características de mensuração a nível ordinal, para descritores de dor;

2. Identificar, dentre os descritores de dor levantados na elaboração do instrumento, os de maior e os de menor atribuição na caracterização da dor pós-operatória.

\section{MATERIAIS E MÉTODOS}

O trabalho compreendeu duas etapas. $\mathrm{Na}$ primeira, construímos um instrumento a nível de mensuração ordinal, contendo descritores de dor. $\mathrm{Na}$ segunda, aplicamo-no com o propósito de identificar os descritores de maior e os de menor atribuição na caracterização da dor pós-operatória.

\subsection{Primeira etapa: elaboração do instrumento de descritores de dor}

\subsubsection{Seleção dos descritores}

Os descritores que compuseram nosso instrumento foram selecionados mediante tradução, para o português, de alguns descritores contidos no Questionário para Dor de McGill (MPQ) (MELZACK, 1975) auxiliados por um dicionário InglêsPortuguês (MICHAELIS, 1992) e uma professora de Inglês; identificação de descritores, a partir de nossa experiência, enquanto enfermeiras, com pacientes que sentiram dor no pós operatório e levantamento de palavras, através de livros, artigos científicos específicos e dicionários da Língua Portuguesa (FERREIRA, 1986) e Médico (HOERR \& OSOL, 1970).

Uma vez selecionados os descritores foram definidos, segundo os dicionários Médico e da Língua Portuguesa, citados anteriormente. Sob cada definição foram colocadas 7 categorias, graduadas de 0 (zero) a 6 (seis), descritas posteriormente, nas quais os participantes deveriam fazer julgamentos, atribuindo escores que representassem o quanto, em sua opinião, tal descritor caracterizava a dor pós-operatória.

Instruções foram elaboradas, por escrito, visando a orientação dos participantes quanto às tarefas a serem cumpridas.

\subsubsection{Validação aparente de conteúdo do instrumento}

\section{Participantes}

Seis profissionais, docentes de universidades federais e estaduais, atuantes em hospitais gerais de médio e de grande porte, dos quais dois eram médicos, dois enfermeiros, um psicólogo e um fisioterapeuta, foram convidados para atuarem como juizes no processo de validação aparente e de conteúdo do instrumento elaborado. Todos possuíam experiência no manejo de pacientes com dor pós-operatória.

\section{Procedimento}

Mediante entrevistas individuais entregamos o instrumento e os orientamos, por escrito, sobre a tarefa a realizar para validarem o instrumento em sua forma aparente e de conteúdo. Portanto, a tarefa dos participantes consistia em modificar, acrescentar ou retirar quaisquer descritores contidos no instrumento manifestando sua concordância com o que lhes estava sendo apresentado, ou seja, se o vocábulo era pertinente para caracterizar a dor pós-operatória ou não e, se a definição dada traduzia o significado do descritor.

\section{Materiais}

Foram utilizados um bloco de papel contendo instruções específicas aos juizes, com itens relacionados à adequação dos termos utilizados, clareza dos itens, adequação das definições e espaço para quaisquer sugestões que se fizessem necessárias; lista de descritores de dor e caneta.

\subsection{Segunda etapa: aplicação do instrumento contendo descritores de dor}

\subsubsection{Teste piloto}

\section{Participantes}

Participaram desse teste seis profissionais, ou seja três médicos e três enfermeiros, os quais atuavam junto ao paciente pós-operado, em hospitais gerais, de médio e grande porte, do Estado de Minas Gerais (cidade de Uberaba).

\section{Procedimento}

Procedemos a aplicação do instrumento através de entrevistas individuais, mediante entrega do mesmo ao participante para que pudesse lê-lo e respondê-lo, após receber orientações escritas sobre a tarefa a ser executada. A tarefa do sujeito consistiu em julgar o grau de atribuição de cada descritor referente à sua utilização na caracterização da dor pós-operatória. Foram utilizadas categorias numéricas que variaram de 0 (zero) a 6 (seis), sendo que a nota 0 (zero) representou o menor grau de atribuição que o descritor poderia receber, a nota 6 (seis) 
o maior e as notas 1 (um), 2 (dois), 3 (três) e 4 (quatro) os graus intermediários.

\section{Materiais}

Para a realização do teste-piloto foi utilizado um bloco contendo instruções específicas aos participantes, uma lista de 119 descritores de dor e caneta.

\subsubsection{Seleção dos descritores de maior e de menor atribuição na caracterização da dor pós-operatória}

\section{Participantes}

Participaram dessa etapa 116 enfermeiros, os quais atuavam em hospitais de médio e grande porte e em escolas de graduação em enfermagem do Estado de São Paulo (cidades de São Paulo, Ribeirão Preto, São José do Rio Preto, Campinas, São Carlos, Ibaté) e do Estado de Minas Gerais (Belo Horizonte, Uberlândia, Uberaba).

O critério para inclusão dos profissionais na amostra foi que estivessem trabalhando junto ao paciente pós-operado, quer em centros de recuperação anestésica, quer em unidades de terapia intensiva, quer em enfermarias cirúrgicas.

\section{Procedimento}

Os participantes foram contatados através de entrevistas individuais, durante as quais o instrumento foi apresentado e os mesmos orientados mediante instruções escritas, quanto à tarefa a ser executada. Esta consistiu em julgar o grau de atribuição de cada descritor na caracterização da dor pós-operatória, através do método de estimação em categorias, já descrito no teste piloto.

Os instrumentos foram entregues aos participantes em mãos, diretamente ou através de docentes e enfermeiros responsáveis pela unidade de atuação dos mesmos.

\section{Materiais}

Utilizamos um bloco de papel contendo instruções específicas aos participantes e uma lista de 119 descritores de dor (selecionados a partir da validação aparente e de conteúdo dos 308 descritores iniciais), caneta, microcomputador e o programa EPI-6.

\section{RESULTADOS E DISCUSSÕES}

Os resultados obtidos nesse trabalho foram expostos seguindo as duas etapas da secção Materiais e Métodos. A primeira, referente à elaboração do instrumento para descritores de dor, e, a segunda, à aplicação do mesmo para realização do teste piloto e seleção dos descritores de maior e dos de menor atribuição na caracterização da dor pós-operatória.

\subsection{Elaboração do instrumento de descritores de dor}

\subsubsection{Seleção dos descritores}

Inicialmente, foram selecionados 308 descritores de dor, segundo as fontes citadas na metodologia, dos quais, 65 a partir da tradução inglês-português dos descritores contidos no MPQ (MELZACK, 1975), para os quais encontramos adequação vocabular em nosso idioma. Problemas relacionados à semântica, constituemse, segundo De BENEDITTIS, et al. (1988), em grande obstáculo à iniciativa de se traduzir literalmente os descritores do M.P.Q. Nesse estudo, descritores tais como "wrenching", "scalding", "rasping", "splintting", "flickering", dentre outros, não foram incluídos em nosso instrumento por tal motivo. Também deixamos de incluir os descritores "cold" e "freezing" por tratar-se de palavras utilizadas pelos ingleses somente em casos muito específicos, tais como a dor de dente (MELZACK, 1975). A respeito do descritor "dull", traduzido como vaga, mal delimitada consideramos seu antônimo - localizada.

Soma-se, ainda, os demais 243 descritores selecionados a partir de nossa experiência profissional, da literatura específica e de dicionários de Língua Portuguesa e Médico. Muitos eram sinônimos como clara, evidente e manifesta; brutal, intensa, forte, violenta e insuportável; incômoda, desagradável, desconfortável, importuna e maçante; malvada, perversa e cruel; constante e contínua; vasta, considerável, grande e importante; cansativa, fatigante, enfadonha e estafante; árdega e ardente; detestável e odiosa, dentre outros. Em outros momentos, consideramos também os antônimos como, complicada/descomplicada; estável/instável; contínua/descontínua.

Algumas expressões, tais como: em fisgada, em facada, como agulhada, como choque, que corrói, como ferroada, como punhalada também foram incluídas uma vez que, no cotidiano, as pessoas utilizam-nas para descrever a dor sentida.

Selecionamos um grande número de palavras, com o propósito de conseguirmos maior abrangência possível em relação aos descritores utilizados na caracterização da dor pós-operatória, em nosso meio profissional e cultural.

\subsubsection{Validação subjetiva: aparente e de conteúdo do IDD}

As críticas e sugestões recebidas após o processo de validação aparente e de conteúdo do instrumento de 
descritores de dor levaram-nos a excluir, substituir e acrescentar descritores em nossa listagem inicial com o propósito de torná-lo mais claro e mais pertinente ao propósito do estudo. Alteramos também, algumas de suas definições, as quais serão apresentadas no final desse item.

Dos 308 descritores contidos inicialmente em nosso instrumento, 190 foram eliminados; considerados pouco adequados para descrever a dor aguda tais como: escravizadora, maldita, infernal e mutilante; mais adequados para descrever a situação do paciente, como contornável, confusa, desastrosa, abrangente, dentre outros; de uso pouco freqüente no cotidiano, tais como: fulgurante, bestial, férvida e cruciante; por haver sinônimos mais adequados à descrição da dor existente na própria listagem inicial, como cortadora, desconfortante, bastante, ininterrupta. Além da exclusão dos descritores, procedemos a algumas substituições e acrescentamos o descritor moderada.

O instrumento já validado e reformulado constou de 119 descritores de dor, considerados mais adequados à caracterização da dor no pós-operatório. A validação aparente e de conteúdo, embora de caráter apenas subjetivo, expressou grande importância na redução do número de descritores, tornando o instrumento mais objetivo.

\subsection{Aplicação do instrumento de descritores de dor}

\subsubsection{Teste piloto}

A realização do teste-piloto resultou em pequenas alterações às instruções referentes à tarefa a ser realizada pelos participantes da pesquisa. Todos os itens foram devidamente preenchidos, sem dificuldades. Pudemos confirmar nessa ocasião que a redução do número de descritores, após a validação do instrumento, fora de vital importância à sua aplicabilidade uma vez que, mesmo em sua forma reduzida, alguns profissionais utilizaram cerca de trinta minutos para preenchê-lo, afirmando tratar-se de tarefa cansativa dado ao grande número de descritores a serem julgados. Uma vez reformulado, à luz das sugestões feitas pelos profissionais que participaram da validação aparente e de conteúdo e do teste-piloto o instrumento foi aplicado com o propósito de selecionarmos os descritores de maior e os de menor atribuição na caracterização da dor pós operatória.

\subsubsection{Seleção dos descritores de maior e menor atribuição na caracterização da dor pós-operatória}

A partir dos escores atribuídos aos 119 descritores de dor, contidos em nosso instrumento, foram calculados a média aritmética e o desvio-padrão com o propósito de selecionarmos os descritores de maior e os de menor atribuição na caracterização da dor pós-operatória.
Nas Tabelas 1 e 2 podemos observar os 15 descritores de maior e os 15 de menor atribuição, respectivamente, na caracterização dessa dor.

Tabela 1 - Descritores de maior atribuição na caracterização da dor pós-operatória, as médias aritméticas (MA), em ordem decrescente e o respectivo desvio-padrão (DP)

\begin{tabular}{l|c|c}
\hline DESCRITORES & $\begin{array}{c}\text { MÉDIA } \\
\text { ARITMÉTICA } \\
\text { (MA) }\end{array}$ & $\begin{array}{c}\text { DESVIO-PADRÃo } \\
\text { (DP) }\end{array}$ \\
\hline intensa & 5,224 & 1,266 \\
\hline forte & 5,155 & 1,169 \\
\hline aguda & 5,069 & 1,270 \\
\hline insuportáve1 & 5,043 & 1,315 \\
\hline contínua & 5,000 & 1,463 \\
\hline que perturba & 4,983 & 1,549 \\
\hline localizada & 4,948 & 1,356 \\
\hline profunda & 4,793 & 1,424 \\
\hline latejante & 4,767 & 1,416 \\
\hline como agulhada & 4,739 & 1,364 \\
\hline desconfortável & 4,698 & 1,578 \\
\hline incômoda & 4,647 & 1,482 \\
\hline desagradáve1 & 4,647 & 1,534 \\
\hline que queima & 4,534 & 1,535 \\
\hline duradoura & 4,457 & 1,506 \\
\hline
\end{tabular}

Dos descritores de maior atribuição na caracterização da dor pós-operatória foram intensa $(\mathrm{MA}=5,224 ; \mathrm{DP}=1,266)$ e forte $(\mathrm{MA}=5,155 ; \mathrm{DP}=1,169)$ sendo, portanto, considerados como os que melhor caracterizam a dor pós-operatória na opinião dos participantes da pesquisa.

Tabela 2 - Descritores de menor atribuição na descrição da dor pós-operatória com as médias aritméticas (MA), em ordem crescente e o respectivo desvio-padrão (DP)

\begin{tabular}{l|c|c}
\hline DESCRITORES & $\begin{array}{c}\text { MÉDIA } \\
\text { ARTMÉTICA } \\
\text { (MA) }\end{array}$ & $\begin{array}{c}\text { DESVIO-PADRÄO } \\
\text { (DP) }\end{array}$ \\
\hline formidáve1 & 1,422 & 1,952 \\
\hline vagarosa & 1,809 & 1,544 \\
\hline circular & 1,862 & 1,788 \\
\hline assaltante & 1,878 & 1,897 \\
\hline circulante & 2,026 & 1,737 \\
\hline destruidora & 2,086 & 1,954 \\
\hline aniquiladora & 2,224 & 2,060 \\
\hline desenfreada & 2,224 & 2,060 \\
\hline que remoe & 2,224 & 2,048 \\
\hline insignificante & 2,233 & 2,144 \\
\hline parada & 2,284 & 1,928 \\
\hline feia & 2,302 & 2,052 \\
\hline miseráve1 & 2,345 & 2,334 \\
\hline que castiga & 2,345 & 2,077 \\
\hline desumana & 2,379 & 2,193 \\
\hline
\end{tabular}


Como podemos observar na Tabela 2, dentre os descritores de menor atribuição, formidável ( $\mathrm{MA}=1,422$ : $\mathrm{DP}=1,952)$ e vagarosa $(\mathrm{MA}=1,809 ; \mathrm{DP}=1,544)$ ocuparam a primeira e segunda posições, respectivamente. Face ao exposto, podemos considerá-los como aqueles descritores que menos caracterizam a dor pós operatória.

\section{CONSIDERAÇÕES FINAIS}

Os resultados obtidos da aplicação do instrumento, aos enfermeiros participantes desse estudo, permitiram-nos concluir que, em nosso meio profissional, os adjetivos de maior atribuição na caracterização da dor pós-operatória foram: intensa, forte, aguda, insuportável, contínua, que perturba, localizada, profunda, latejante, como agulhada, desconfortável, incômoda, desagradável, que queima e duradoura; os de menor atribuição foram: formidável, vagarosa, circular, assaltante, circulante, destruidora, aniquiladora, desenfreada, que remoe, insignificante, parada, feia, miserável, que castiga e desumana. Em relação aos descritores de maior atribuição na caracterização dessa dor observamos que a maioria pertence ao grupo sensorial e avaliativo da experiência dolorosa, preconizados por MELZACK \& TORGERSON (1971). Quanto aos descritores de menor atribuição, muitos pertencem à dimensão afetiva da dor, o que está de acordo com os achados de MELZACK et al. (1987) e BÉLANGER et al. (1989) os quais, utilizando o MPQ (MELZACK, 1975) para mensurar a dor no período pós-operatório, observaram que os descritores escolhidos com menor freqüência pelos pacientes eram do grupo afetivo. Todavia, uma comparação direta não é possível, uma vez que não há outros estudos nos quais tenha sido feito elaboração de escalas de descritores específicos para a dor pós-operatória.

Nesse contexto, o presente estudo foi desenvolvido possibilitando a elaboração de um instrumento de descritores de dor e a identificação daqueles de maior e de menor atribuição referentes à descrição da dor pós-operatória na opinião de enfermeiros. Após refinamento, o mesmo será aplicado no período pós-operatório, a médicos e pacientes, sendo para os últimos em forma reduzida, com o propósito não só de avaliação dessa dor mas, também, de validá-lo.

Quanto à tradução literal dos descritores oriundos da Língua Inglesa ressaltamos os problemas relacionados à semântica, maior obstáculo à adequação dos termos em nosso idioma. Tal hipótese, enfocada também por estudos cujos objetivos se assentaram na conquista de uma versão para o MPQ (original do inglês), em outros idiomas, poderia levar o paciente a escolher descritores pouco apropriados para descrever a dor sentida, em detrimento de outros, mais utilizados em sua língua, porém, ausentes da listagem apresentada (PONTINËN \& KETOVUORI, 1983; RADVILA et al., 1987; De BENEDITTIS et al., 1988).

Compreendemos que o desenvolvimento da linguagem é uma etapa fundamental na evolução do controle, logo, a descrição verbal da intensidade da dor e de suas qualidades, feita pelos profissionais e indivíduos que a vivenciam, é de fundamental importância para sua mensuração. Evidentemente, conhecer a linguagem utilizada nessa descrição (os descritores de dor) e compreender aquilo que está sendo comunicado é essencial aos profissionais da área, cujo objetivo é o controle da dor referida pelo próprio paciente.

Estamos certos de que este trabalho constituiuse em primeiro e importante passo para a elaboração de instrumentos multidimensionais, os quais possibilitarão identificar as dimensões envolvidas nos relatos de dor dos pacientes e refletir na prática assistencial de enfermagem, uma vez que, a mensuração da dor clínica, direcionará a tomada de decisões de profissionais da área especialmente em relação ao emprego de medidas terapêuticas que visem adequado alívio, impedindo que o controle da dor pós-operatória seja embasado apenas nos pressupostos dos profissionais da área. Ressaltamos sua relevância para o ensino em enfermagem, pois alertanos para oferecermos ao aluno de graduação condições suficientes à formação de uma visão mais abrangente e crítica sobre a dor no pós-operatório. Enfatizamos sua contribuição à pesquisa em enfermagem, cujos estudos metodológicos e de mensuração em níveis mais precisos se fazem essenciais.

Pretende-se ainda, a partir desse estudo, em projeto mais amplo, o qual consistirá de outros métodos, tais como: estimação de magnitudes e estimação de comprimento de linhas, validar os descritores de maior atribuição à caracterização da dor pós-operatória e averiguar os descritores mais utilizados para descrever a dor sentida após cada tipo de cirurgia. Para detalhes sobre a metodologia a ser aplicada posteriormente ver FALEIROS SOUSA (1993) e FALEIROS SOUSA \& da SILVA (1996).

\section{CATEGORY ESTIMATION OF THE POSTOPERATIVE PAIN DESCRIPTORS}

In this study we investigate the language used by nurses in the characterization of postoperative pain. In order to do this, an instrument was elaborated containing initially 308 pain descriptors, which were studied as to their apparent and content validity. After this study, using 119 descriptors, the instrument was applied to 116 nurses. The category estimation method was used, with scores varying from 1 to 7. The mean score and standard deviation were calculated for the scores 
obtained. The descriptors of highest ascriptions were, in a decreasing order: intense, strong, acute, unbearable and continuous; those of lower ascriptions were: circulating, assailing, circular, slow and formidable.

KEY WORDS: category estimation, postoperative pain, pain measurement

\section{ESTIMACIÓN EN CATEGORÍAS DE DESCRIPTORES DEL DOLOR POSTQUIRÚRGICO}

En este estudio investigamos el lenguaje utilizado por enfermeros en la caracterización del dolor postquirúrgico. Para esto, fue elaborado un instrumento conteniendo, inicialmente, 308 descriptores de dolor, los cuales fueron analizados en su validad aparente y de contenido. Después de ese análisis, con 119 descriptores, el instrumento fue aplicado a 116 enfermeros. Se utilizó el método de estimación en categorías con escores variando de 1 a 7. Fueron calculados la media aritméctica y la desviación estándar de los escores obtenidos. Los descriptores de mayor atribución, en orden decreciente, fueron: intensa, fuerte, aguda, insoportable y contínua; los de menor atribuición fueron: circulante, punzante, lento y formidable.

TÉRMINOS CLAVES: estimación en categorías, dolor postquirúrgico, medición del dolor

\section{REFERÊNCIAS BIBLIOGRÁFICAS}

01. BÉLANGER, E.; MELZACK, R.; LAUZON, P. Pain of first-trimester abortion: a study of psychosocial and medical predictors. Pain, v.36, p.339-350, 1989.

02. BOUlAnGER, A. et al. Comparison between patient-controlled analgesia and intra-muscular meperidine after thoracotomy. Canadian Journal Anaesthesia, v. 40, n. 5, p.409 - 415, 1993.

03. BOUREAU, F.; LUU, M.; DOUBRÈRE, J.F. Comparative study of the validity of four french McGill pain questionnaire (M.P.Q.) versions. Pain, n.50, p.59-65, 1992.

04. CAMP, L. D.; O’SULLIVAN, P. S. Comparison of medical, surgical and oncology patients' descriptions of pain and nurses' documentation of pain assesments. Journal Advanced Nursing, v.12, p. 593-598, 1987.

05. CARTWRIGHT, P.D. Pain control after surgery: a survey of current practice. Annals Royal College Surgeons England, v.67, p.13-16, 1985.

06. CHOINIÈRE, M. et al. Comparisons between patients' and nurses' assessment of pain and medication efficacy in severe burn injuries. Pain, v. 40, p. 143-152, 1990.

07. CONSTANT, I.; BONNET, F. Consequências fisiopatológicas da dor pós-operatória. In: BONNET, F. (ed.). A dor no meio cirúrgico. Porto Alegre: Artes Médicas, 1993. p.71-84.

08. DAVITZ, L.J.; PENDLENTON, S.H. Nurses inferences of suffering. Nursing Research, v.18, p.100-107, 1969.

09. De BENEDITTIS, G. et al. The italian pain questionnaire. Pain, v.33, p.53-62, 1988.
10. DONOVAN, M.; DILION, P.; MCGUIRRE, L. Incidence and characteristics of pain in a sample of medical-surgical inpatients. Pain, v.30,p.69-78, 1994.

11. EISENACH, J.C. Incidência da dor pós-operatória e fatores predisponentes. In: BONNET, F. (ed.) A dor no meio cirúrgico. Porto Alegre: Artes Médicas, 1993. p.48-56.

12. FALEIROS SOUSA, F.A.E. Prestígio profissional do enfermeiro: um enfoque da psicofísica social. Ribeirão Preto, 1993. 175p. Tese (doutorado) Escola de Enfermagem de Ribeirão Preto, Universidade de São Paulo.

13. FALEIROS SOUSA, F.A.E.; da SILVA, J.A. Uso e aplicação da metodologia psicofísica na pesquisa em enfermagem. Revista Latino Americana Enfermagem, v.4, n.2, p.147-178, 1996.

14. FERREIRA, A.B.H. Novo dicionário Aurélio da língua portuguesa. 2.ed. Rio de Janeiro: Nova Fronteira, 1986.

15. FORTUNA, A.; FORTUNA, A.O. Dor pósoperatória. Revista Brasileira Cirurgia, v.77, n. 5, p.291-294, 1987.

16. GREENWALD, H.P. Interethnic differences in pain perception. Pain, v.14, p.157-163, 1991.

17. HARDWICK, R. H.; DURDEY, P. Should rubber band ligation of haemorrhoids be performed at the initial outpatient visit? Annals Royal College Surgeon England, v.76, p.185-187, 1994.

18. HARRISON, A. Arabic pain words. Pain, v.32, p. 239-250, 1988.

19. HOERR, N.L.; OSOL, A. (eds.). Dicionário médico ilustrado Blakiston. São Paulo: Andrei, 1970.

20. HOLM, K. et al. Effect of personal pain experience on pain assessment. Image: Journal Nursing Scholarship, v.21, n.2, p.72-75, 1989. 
21. KATZ, J. et al. Pre-emptive lumbrar epidural anaesthesia reduces postoperative pain and patient-controlled morphine consumption after lower abdominal surgery. Pain, v.59, p.395-403, 1994.

22. KIM, H. E. et al. Developing a translation of the McGill pain questionnaire for cross-cultural comparison: an example from norway. Journal Advanced Nursing, v.21, p.421- 426,1995.

23. LENBURG, C.B.; GLASS, H.P.; DAVITZ, L.J. Pain in relation to the perceiver. Nursing Reseach, v.19, p.392-398, 1970.

24. LÒPES, R.R.; FERRER, I.; PAGEROLS, M. The spanish pain questionnaire. Pain, v.5, p. 304, 1990. Suppl.

25. MAIANI, G.; SANAVIO, E. Semantics of pain in Italy: the italian version of the McGILL pain questionnaire. Pain, v.22, p.399-405, 1985.

26. MARKS, R.M.; SACHAR, E.J. Undertreatment of medical inpatients with narcotic analgesics. Annals Internal Medicine, v.78, n.2, p.173-181, 1973.

27. MARTIN, B.A.; BELCHER, J.V. Influence of cultural background on nurses attitudes and care of the oncology patient. Cancer Nursing, v.9, n.5, p.230-237, 1986.

28. McCAFFERY, M. Understanding your patients' pain. Nursing, v.10, n.9, p.26-31, 1980.

29. McCAFFERY, M.; HART, L.L. Undertreatment of acute pain with narcotics. American Journal Nursing, v.76, n.10, p.1586-1591, 1976.

30. MELZACK, R. The McGill pain questionnaire: major properties and scoring methods. Pain, v.1, p.277299, 1975.

31. MELZACK, R.; TORGERSON, W.S. On the language of pain. Anesthesiology, v.34, n.1, p. 50-59, 1971.

32. MELZACK, R.; WALL, P.D.; TY, T. C. Acute pain in an emergency clinic: latency of onset and descriptor patterns related to different injuries. Pain, v.14, p. 33-43, 1982.

33. MELZACK, R. et al. Pain on a surgical ward: a survey of the duration and intensity of pain and the effectiveness of medication. Pain, v.29, p.67$72,1987$.

34. MICHAELIS, H. Dicionário inglês-português. São Paulo: Melhoramentos, 1992.
35. OWEN, H.; MCMILLAN, V.; ROGOWSKI, D.

Postoperative pain therapy: a survey of patients' expectations and their experiences. Pain, v.41, p.303-307, 1990.

36. PIMENTA, C.A.M. et al. Dor: ocorrência e evolução no pós-operatório de cirurgia cardíaca e abdominal. Revista Paulista Enfermagem, v.11, n.1, p.3-10,1992.

37. PÖNTINEN, P.J.; KETOVUORI, H. Verbal measurement in non-english language: the finnish pain questionnaire. In: MELZACK, R. Pain measurement and assessment. New York: Raven Press, 1983. p.85-93.

38. RADVILA, A. et al. The development of a German language (Berne) pain questionnaire and its application in a situation causing acute pain. Pain, v.28, p.185-195,1987.

39. READING, A.E. A comparison of the McGill pain questionnaire in chronic and acute pain. Pain,v.13, p.185-192, 1982.

40. READY, L.B.; EDWARDS, W.T. (eds.) Tratamento da dor aguda. Rio de Janeiro: Revinter, 1995.

41. SCHULTE-STEINBERG, H. et al. Intraperitoneal versus interpleural morphine or bupivacaine for pain after laparoscopic cholecystectomy. Anesthesiology, v.82, p.634 - 640, 1995.

42. STEIN, C.et al. Analgesic effect of intraarticular morphine after arthroscopic knee surgery. New England Journal Medicine, v.325, n.16, p.1123 - 1126, 1991.

43. STRAND,L.I.; WISNES, A. R. The development of a norwegian pain questionnaire. Pain, v.16, p.6166, 1991

44. TESKE, K.; DAUT, R.L.; CLEELAND, C.S. Relationships between nurses' observations and patients' self-reports of pain. Pain, v.16, p.289296,1983.

45. THOMAS, V. et al. Psychological characteristics and the effectiveness of patient-controlled analgesia. British Journal Anaesthesia, v. 74, p. 271-276, 1995.

46. VANDERIET, K.et al. The McGill pain questionnaire constructed for the dutch language (MPQ-DV) Preliminary data concerning reliability and validity. Pain, v.30, p.395-408, 1987.

47. WEIS, O.F. et al. Attitudes of patients, housestaff, an nurses toward postoperative analgesic care. Anesthesia Analgesia, v. 62, p. 70-74,1983.

48. ZALON, M.L. Nurses' assessment of postoperative patients' pain. Pain, v. 54, p. 329-334, 1993. 